\title{
Análisis de idoneidad didáctica sobre la geometría espacial en el currículo nacional intercultural bilingüe de Ecuador
}

\author{
Andrea Cruz Verdugo \\ Universidad de Granada, Espańa. \\ anddrea25@correo.ugr.es \\ María Eugenia Reyes Escobar \\ Corporación Municipal de Puente Alto, Chile.
}

Recibido: 9 - febrero-2018 / Aceptado: 31 - julio - 2018

\section{Resumen}

Se presenta el uso de la Noción de Idoneidad Didáctica, específicamente la Guía de Valoración (GVID) (Godino, 2013) compuesta por un sistema de indicadores o criterios adaptados a la geometría espacial por Cruz, Gea y Giacomone (2017). Se presenta la aplicación de esta herramienta en el análisis epistémico del currículo de Educación Intercultural Bilingüe (EIB) Kichwa, para esclarecer si su contenido matemático sobre la visualización espacial tridimensional en los primeros niveles educativos contiene elementos necesarios para promover un aprendizaje idóneo. Se realizan sugerencias de mejora, lo que podría ayudar a alcanzar niveles altos de idoneidad en los procesos instruccionales.

Palabras clave: idoneidad didáctica, geometría espacial, educación intercultural. 


\begin{abstract}
In this study the concept of Didactical Suitablity is used, refering to the assessment Guidance (GVID) (Godino, 2013) composed by a system of indicators or criteria adapted to the Spatial Geometry by Cruz, Gea y Giacomone (2017). The application of this tool is presented in the epistemic analysis of the curriculum from Bilingual Intercultural Education (EIB) Kichwa, to clarify if its mathematical content about the three-dimensional spatial display in the first educational levels has necessary elements to promote a suitable learning. Suggestions are made in order to help reaching high levels of suitability in the instructional processes.
\end{abstract}

Keywords: suitability, didactics, spatial geometry, intercultural education. 


\section{Introducción}

$\mathrm{E}$ 1 Ministero de Educación del Ecuador proporciona al sistema educativo los currículos nacionales interculturales bilingües (MinEduc, 2017) los que han sido conformados a partir del ajuste curricular del año 2016 para fomentar el desarrollo intercultural en los procesos de enseñanza y aprendizaje. Estas herramientas curriculares son pertinentes a las culturas y lenguas de los pueblos nacionales e indígenas del país, y se emplearán obligatoriamente en el Sistema de Educación Intercultural Bilingüe (SEIB), por lo que se han organizado en base al proceso educativo del Modelo del Sistema de Educación Intercultural Bilingüe (MOSEIB): Educación Infantil Familiar Comunitaria - EIFC; la Educación Básica Intercultural Bilingüe conformada por: Inserción al Proceso Semiótico - IPS, Fortalecimiento Cognitivo, Afectivo y Psicomotriz - FCAP, Desarrollo de Destrezas y Técnicas de Estudio - DDTE, Proceso de Aprendizaje Investigativo - PAI, y, por último, el Bachillerato (MinEduc, 2017, pág. 4).

En el programa propuesto se plantean los Saberes y Conocimientos que "se refieren a las destrezas con criterio de desempeño que tiene el currículo nacional, de manera que estos Saberes aciertan con los definidos a nivel nacional" (MinEduc, 2017, pág. 124). También se plantean los Dominios de aprendizaje, siendo un aporte específico al currículo nacional y son entendidos como la "capacidad de aplicar a la realidad los conocimientos adquiridos y poder explicarlos" (MinEduc, 2017, pág. 5). Específicamente en la educación matemática, se pretende incorporar en forma inicial las necesidades personales y sociales de uso del número, el aprendizaje de los conceptos lógico-matemáticos y su aplicación en las resolución de problemas de la vida cotidiana (MinEduc, 2017, pág. 35).

Este trabajo está basado en la noción de Idoneidad Didáctica (ID) (Godino, 2013) específicamente la faceta epistémica de la Guía de Valoración de Godino (2013), que es adaptada por Cruz, Gea y Giacomone (2017) en un sistema de indicadores, criterios o heurísticas específicos sobre la geometría espacial.

Su propósito es mostrar la aplicación de este sistema en el análisis y reflexión de los significados institucionales pretendidos (Saberes y Conocimientos, Dominios) y referidos al desarrollo de la visualización de figuras de dos (2D) y tres dimensiones (3D). Con el análisis se pretende observar el grado de representatividad que tienen dichos significados respecto a un significado de referencia en términos de las situaciones-problema, los elementos lingüísticos y las representaciones usadas; así también los elementos conceptuales, proposicionales, procedimentales y argumentativos puestos en juego en la propuesta curricular intercultural. Con la reflexión, en cambio, esclarecer si el contenido didáctico-matemático que se plantea a nivel elemental, contiene elementos necesarios para promover un aprendizaje óptimo y a la vez, presentar sugerencias didácticas para ayudar a alcanzar niveles altos de idoneidad en los procesos instruccionales. 


\section{Metodología}

En este estudio se utiliza una metodología cualitativa de tipo descriptiva (Bisquerra, 1989) para analizar los contenidos didáctico-matemáticos presentes en las directrices curriculares ecuatorianas para los primeros cursos de la Educación Básica (Preparatoria y Elemental de entre 5 y 8 ańos), específicamente en los Saberes, Conocimientos y Dominios sobre la visualización de figuras 2D y 3D del currículo de EIB Kichwua (MinEduc, 2017). Para esto, se hace uso de los criterios de la dimensión epistémica que fueron especificados y adaptados a la geometría espacial por Cruz et al. (2017), los que son presentados en la Tabla 1.

Tabla 1. Adaptación de los componentes e indicadores de idoneidad epistémica de Godino (2013) por Cruz, Gea y Giacomone (2017, pp. 7-9)

\begin{tabular}{|c|c|}
\hline Componentes & Criterios \\
\hline Situaciones-problemas & $\begin{array}{l}\text { Criterios especificos: } \\
\text { Se deberían abordar problemas que involucren la interacción con las } \\
\text { formas en el espacio, aplicando aspectos intuitivos y prácticos que poco } \\
\text { a poco se vayan formalizando, tales como: } \\
\text { - Reconocer y relacionar los atributos de las formas. } \\
\text { - Coordinar e integrar vistas de objetos. } \\
\text { - Plegar y desplegar desarrollos. } \\
\text { - Identificar partes y elementos de un sólido. } \\
\text { - Componer y descomponer en partes un objeto tridimensional. } \\
\text { - Rotar un objeto en el espacio. }\end{array}$ \\
\hline Lenguajes & $\begin{array}{l}\text { Criterios especificos: } \\
\text { - Distinguir y describir las representaciones verbales, planas, gráficas y } \\
\text { de modelos manipulables. } \\
\text { - Nombrar y describir formas tridimensionales y su posición en el } \\
\text { espacio con relación a otros objetos y personas. } \\
\text { - Describir la orientación y la estructura de un objeto tridimensional. } \\
\text { - Utilizar diferentes términos para la descripción de posición, dirección } \\
\text { y distancia de los objetos en el espacio: arriba, abajo, delante, detrás, } \\
\text { derecha, izquierda, cerca, lejos, etc. }\end{array}$ \\
\hline
\end{tabular}




\begin{tabular}{|c|c|}
\hline $\begin{array}{l}\text { Reglas (Definiciones, } \\
\text { propiedades, } \\
\text { procedimientos) }\end{array}$ & $\begin{array}{l}\text { Criterios especificos: } \\
\text { Se procura que los estudiantes aprendan a distinguir en los objetos } \\
\text { físicos, representaciones y diagramas de las correspondientes figuras } \\
\text { geométricas, como entidades o formas no ostensivas cuyo uso está } \\
\text { determinado por las reglas que los definen. En particular, } \\
\text { - Dominar e identificar las propiedades de las figuras para utilizarlas en } \\
\text { el proceso deductivo y realizar argumentaciones. } \\
\text { - Reconocer figuras que tengan atributos específicos, tales como un } \\
\text { número dado de ángulos o un número dado de caras iguales. } \\
\text { - Distinguir y comparar los atributos que definen y no definen a las } \\
\text { figuras tridimensionales. } \\
\text { - Clasificar figuras de tres dimensiones de acuerdo con sus propiedades } \\
\text { y características. } \\
\text { - Construir y desarmar objetos tridimensionales dadas ciertas vistas } \\
\text { ortogonales y desarrollos planos, respectivamente. } \\
\text { - Desarrollar habilidades de visualización y razonamiento espacial, a } \\
\text { través de los procedimientos utilizados en la realización de una tarea. }\end{array}$ \\
\hline Argumentos & $\begin{array}{l}\text { Criterios especificos: } \\
\text { Se procura que los estudiantes comprendan la necesidad y utilidad de } \\
\text { construir demostraciones deductivas de proposiciones, que admiten } \\
\text { una representación visual que aparentemente se hace necesaria. En } \\
\text { particular, } \\
\text { - Aplicar una transición progresiva de las argumentaciones desde los } \\
\text { significados intuitivos e informales de los conceptos geométricos a sus } \\
\text { respectivas formalizaciones. } \\
\text { - Formular y comprobar conjeturas sobre propiedades geométricas y sus } \\
\text { relaciones. } \\
\text { - Desarrollar argumentos lógicos para justificar conjeturas y conclusiones } \\
\text { de forma gradual de acuerdo al nivel. }\end{array}$ \\
\hline Relaciones & $\begin{array}{l}\text { Criterio especifico: } \\
\text { Se procura la conexión de las ideas matemáticas, específicamente las } \\
\text { geométricas espaciales, para ser presentadas, reconocidas y enseñadas } \\
\text { como un todo organizado en contextos no matemáticos: } \\
\text { - Reconocer, identificar, distinguir, definir, clasificar, razonar y relacionar } \\
\text { figuras tridimensionales en el entorno. }\end{array}$ \\
\hline
\end{tabular}




\section{Saberes y conocimientos, dominios}

La extracción de Unidades, Saberes y Conocimientos, y Dominios que se presentan en las tablas 2 y 3, son los referidos a la geometría espacial (MinEduc, 2017) y propuestos para ser abordados durante los primeros cuatro grados de la enseńanza básica $\left(1^{\circ}\right.$ a $\left.4^{\circ}\right)$.

Tabla 2. IPS: Inserción al Proceso Semiótico, $1^{\circ}$

\begin{tabular}{lll}
\hline UNIDADES & SABERES Y & DOMINIOS \\
& CONOCIMIENTOS &
\end{tabular}

UNIDAD 11

"Somos hijos e hijas del cosmos"

$$
\text { OBJETIVO: }
$$

Asumir con responsabilidad que somos parte de la madre naturaleza y del cosmos; a través del conocimiento y valoración practicada en la nacionalidad, para garantizar una convivencia armónica y responsable con las formas de vida.
Noción de ubicación espacial: arriba/abajo, delante/atrás y cerca/lejos de seres del entorno.

demás.

Noción de tamaño: grande, mediano y pequeño.

Derecha, izquierda.

\begin{abstract}
Reconoce la ubicación de objetos del entorno según las nociones de arriba/ abajo, adelante/atrás y cerca/lejos.
\end{abstract}

Describe objetos del entorno utilizando nociones de longitud: alto/bajo, largo/corto.

Reconoce la posición de objetos del entorno: derecha, izquierda.

Reconoce la derecha e izquierda en los demás.

Reconoce, estima y compara objetos de acuerdo a su tamańo. 
UNIDAD 12

"Nuestra familia"

OBJETIVO:

Ejercer los derechos y obligaciones como persona e integrante de la familia, a través de la comprensión de roles $y$ funciones en el contexto familiar y comunitario.
Derecha e izquierda en los demás.

Color, tamaño, longitud de objetos del entorno.

Colecciones de objetos.

Nociones y relaciones espaciales con referencia a sí mismo (entre, alrededor).

Cuerpos geométricos y figuras en elementos del entorno.

Figuras geométricas.

Noción de ubicación espacial: encima /debajo de objetos del entorno en situaciones del entorno.
Reconoce la derecha e izquierda en los demás.

Reconoce las semejanzas y diferencias entre los objetos del entorno de acuerdo a su forma y sus características físicas (color, tamaño y longitud).

Agrupa colecciones de objetos del entorno según sus características físicas: color, tamaño (grande/pequeño), longitud (alto/bajo y largo/corto).

Distingue las principales nociones $y$ relaciones espaciales con referencia a sí mismo (entre alrededor).

Identifica las figuras geométricas en objetos del entorno.

Establece semejanzas $y$ diferencias entre objetos del entorno y cuerpos geométricos.

Reconoce figuras geométricas (triángulo, cuadrado, rectángulo y círculo) en objetos del entorno.

Reconoce la ubicación de objetos del entorno según las nociones de encima debajo. 


\begin{tabular}{|c|c|c|}
\hline $\begin{array}{l}\text { UNIDAD } 13 \\
\text { "Nuestra comunidad/ El Centro } \\
\text { Educativo Comunitario }\end{array}$ & $\begin{array}{l}\text { Noción de conjunto utilizando } \\
\text { elementos del entorno. }\end{array}$ & $\begin{array}{l}\text { Recolecta y } \\
\text { agrupa objetos } \\
\text { de acuerdo a sus } \\
\text { atributos y establece }\end{array}$ \\
\hline $\begin{array}{l}\text { Intercultural Bilingüe - CECIB” } \\
\text { OBJETIVO: }\end{array}$ & $\begin{array}{l}\text { Clasificación, composición, } \\
\text { ordenación, seriación con } \\
\text { elementos del entorno. }\end{array}$ & comparaciones. \\
\hline $\begin{array}{l}\text { Posibilitar una mejor convivencia en } \\
\text { la comunidad educativa, mediante el } \\
\text { aprendizaje significativo de diversos } \\
\text { medios de comunicación, transporte y } \\
\text { TIC para establecer un criterio propio }\end{array}$ & & $\begin{array}{l}\text { Clasifica objetos } \\
\text { de acuerdo a sus } \\
\text { características. }\end{array}$ \\
\hline acerca de la realidad local. & & $\begin{array}{l}\text { Describe y reproduce } \\
\text { patrones con objetos } \\
\text { del entorno por } \\
\text { color, forma, tamaño, } \\
\text { longitud o con } \\
\text { siluetas de figuras } \\
\text { geométricas, sonidos } \\
\text { y movimientos. }\end{array}$ \\
\hline
\end{tabular}

Tabla 3. FCAP: Fortalecimiento Cognitivo, Afectivo y Psicomotriz, $2^{\circ}$ a $4^{\circ}$

\begin{tabular}{lll}
\hline UNIDADES & SABERES Y & DOMINIOS \\
& CONOCIMIENTOS & \\
\hline
\end{tabular}

\section{UNIDAD 16}

"Época de preparación de la Madre Tierra”

\section{OBJETIVO:}

Articular los conocimientos y prácticas propias de la comunidad y su entorno, para fortalecer los lazos de identidad socio cultural dentro y fuera del centro educativo.
Conjunto y elemento, relación Discrimina atributos de pertenencia. de los objetos del entorno e identifica elementos que Atributos de objetos del entorno. pertenecen a un conjunto.

Reconoce los atributos de los objetos, describe patrones de objetos del entorno en base a un atributo.

Reproduce patrones de objetos del entorno en base a un atributo. 
UNIDAD 17

"Época de la siembra"

\section{OBJETIVO:}

Fomentar el uso de técnicas ancestrales en la siembra, a través de la vivencia de los diferentes contextos y transmisión de saberes que evidencien la funcionalidad de la lengua oral como herramienta de comunicación a fin de fortalecer los diferentes espacios comunitarios
Conjunto y subconjunto.

Patrones de figuras del entorno y cuerpos geométricos en base a un atributo.

Cuerpos geométricos: pirámides, prismas. Identificación en objetos del entorno.

Propiedades de los cuerpos geométricos.
Representa

gráficamente conjuntos y subconjuntos discriminando las propiedades de los elementos.

Reproduce patrones de objetos del entorno en base a dos atributos.

Reproduce patrones de figuras del entorno y cuerpos geométricos en base a un atributo.

Identifica y compara cuerpos geométricos (pirámide y prismas) en objetos del entorno.

Reconoce y diferencia las propiedades de pirámides y prismas, en objetos del entorno y/o modelos geométricos. 


\section{UNIDAD 18}

"Época de

Crecimiento y cuidado de las plantas"

\section{OBJETIVO:}

Explicar sobre la importancia y el cuidado del entorno natural y cultural, a través de su conocimiento y saberes en el cuidado de las plantas, para garantizar una convivencia entre los seres vivos.
Patrones de figuras del entorno Reproduce patrones y cuerpos geométricos en base de figuras del a dos atributos.

entorno y cuerpos geométricos en base a dos atributos.

Cuerpos geométricos: cono, cilindro y esfera.

Identifica en objetos del entorno cuerpos geométricos como conos, cilindros y esferas.

Propiedades de los cuerpos geométricos.

Reconoce y diferencia las propiedades de conos, cilindros, en objetos del entorno y/o modelos geométricos.

\section{UNIDAD 19}

"Época de Florecimiento"

Clasificación de cuerpos geométricos.

Clasifica los cuerpos geométricos según sus conocimientos.

Figuras geométricas: triángulo, cuadrado, rectángulo y círculo: Reconoce las Identificación en objetos del entorno.

figuras geométricas en elementos del entorno e identifica el lado interior, exterior

Lados, ángulos y vértices $\mathrm{y}$ frontera.

Identifica lados, ángulos y vértices en las siguientes figuras geométricas: triángulo, cuadrado y rectángulo.

Clasifica figuras geométricas según sus propiedades. 
UNIDAD 22

"Organización comunitaria"

\section{OBJETIVO:}

Incentivar la aplicación de saberes ancestrales en salud integral, seguridad alimentaria a nivel local y prevenir enfermedades conocidas y extrańas dentro de la comunidad.

\section{Cuerpos geométricos:}

pirámides, prismas:

Elementos y propiedades.
Identifica y compara cuerpos geométricos "pirámides, prismas" a partir de sus elementos y propiedades.
UNIDAD 23

"Nuestra sabiduría"

\section{OBJETIVO:}

Desarrollar actitudes fundadas en el respeto por la vida, a través de la integración de conocimientos comunitarios sobre el trabajo, liderazgo, y mediación de conflictos, que aporten a la resolución de problemas cotidianos.

UNIDAD 24

"Nuestras fiestas"

\section{OBJETIVO:}

Explicar el saber artístico cultural de su pueblo o nacionalidad, como estrategia para potenciar su autoestima personal e identidad cultural.
Cuerpos geométricos: cono, cilindro y esfera. Elementos y propiedades.
Identifica y compara cuerpos geométricos (cono, cilindro y esfera) a partir de sus elementos y propiedades.
Figuras geométricas "triángulo, Reconoce figuras cuadrado, rectángulo": Elementos y propiedades.

Lados, vértices y ángulos. geométricas (triángulo, cuadrado, rectángulo) a partir de sus elementos y propiedades.
Identifica lados, vértices y ángulos en las figuras geométricas.

Identifica formas y circulares en cuerpos geométricos del entorno y/o modelos geométricos.

Valorar y proteger los espacios y elementos sagrados de su cultura y relacionarlos con la medicina ancestral y farmacológica, para

prevenir enfermedades que atacan a los niños y nińas de la comunidad. 


\begin{tabular}{lll}
\hline UNIDAD 28 & $\begin{array}{l}\text { Semirrecta, segmento y } \\
\text { ángulo. }\end{array}$ & $\begin{array}{l}\text { Reconoce la } \\
\text { semirrecta, segmento, } \\
\text { ángulo y representa } \\
\text { en forma gráfica. }\end{array}$ \\
$\begin{array}{l}\text { OBJETIVO: } \\
\begin{array}{l}\text { Conocer y valorar la medicina natural } \\
\text { practicada en la nacionalidad, mediante } \\
\text { el uso de espacios y elementos de la } \\
\text { madre naturaleza, para dinamizar los } \\
\text { saberes y tradiciones desde la identidad }\end{array}\end{array}$ & \\
individual y colectiva. & & \\
\hline $\begin{array}{l}\text { UNIDAD 29 } \\
\text { "Principios de la Nacionalidad" }\end{array}$ & Líneas paralelas secantes y \\
$\begin{array}{l}\text { OBJETIVO: } \\
\text { secantes perpendiculares. }\end{array}$ & $\begin{array}{l}\text { Reconoce líneas } \\
\text { paralelas, secantes }\end{array}$ \\
$\begin{array}{l}\text { Cultivar en los estudiantes la } \\
\text { reciprocidad, complementariedad y } \\
\text { correspondencia, en la búsqueda del } \\
\text { perfil ideal de una nueva persona con } \\
\text { la sabiduría del pasado, acorde a los } \\
\text { tiempos actuales y con proyección hacia } \\
\text { el futuro. }\end{array}$ & & $\begin{array}{l}\text { perpendiculares, en } \\
\text { objetos del entorno. }\end{array}$ \\
\hline $\begin{array}{l}\text { UNIDAD 33 } \\
\text { "Organización, liderazgo y derechos" }\end{array}$ & de objetos. \\
$\begin{array}{l}\text { OBJETIVO: } \\
\text { ejercer derechos y responsabilidades, } \\
\text { valores y principios comunitarios. }\end{array}$ & & \\
\hline
\end{tabular}

\section{Resultados del análisis y sugerencias}

En este apartado se muestra la aplicación de los indicadores específicos de idoneidad presentados en la Tabla 1 al análisis de los contenidos sobre la geometría espacial mostrados en las Tablas 2 y 3 , incluye sugerencias para su implementación cuando así corresponda:

En el componente Situaciones-problema con los Saberes y Dominios propuestos, se pretende que los estudiantes interactúen con las formas y objetos en el espacio para reconocer, identificar y relacionar su posición, forma y características físicas con 
objetos del entorno. Se propone trabajar tanto con figuras de 2D (triángulo, cuadrado, rectángulo y círculo) como con figuras 3D (pirámide, prisma, cono y cilindro).

Para el logro eficaz de los Dominios propuestos, se encuentra una dificultad, puesto que no se explicita la aplicación de situaciones-problema que consideren en forma gradual los aspectos intuitivos y prácticos. Por esto se sugiere incorporar y abordar, situaciones que involucren el pliegue y despliegue de desarrollos para la coordinación mental en la formación de un objeto 3D como modelo único, la composición y descomposición en partes de una figura 3D y situaciones en que los estudiantes puedan rotar un objeto en el espacio para visualizarlo desde diferentes puntos.

En el componente Lenguaje, se propone el uso de objetos del entorno para describir sus características en cuanto a la longitud, tamaño, ubicación y posición con conceptos específicos (largo, corto, grande, pequeño, izquierda, derecha, arriba, abajo, encima, debajo, adelante, atrás, cerca, lejos). Como también, se pretende que los estudiantes reconozcan e identifiquen las características de los mismos objetos (lados, ángulos, vértices, líneas paralelas y perpendiculares) para compararlos y clasificarlos. Sin embargo, no se explicitan situaciones en que se haga uso de representaciones de las figuras 2D y 3D en forma gráfica y contextualizada. Por tanto, se sugiere incentivar el uso del propio lenguaje (vocabulario informal) para denominar y describir las características que definen y no definen a una figura u objeto. También, la utilización de material gráfico y manipulable atractivos para la localización de objetos y personas a través de elementos orientados para crear oportunidades de observación y exploración de figuras, y facilitar la identificación y comparación en forma idónea.

En el componente Reglas (definiciones, propiedades, procedimientos) es necesario explicitar la importancia de que los estudiantes dominen las definiciones de los conceptos, realizando un proceso de transición progresivo desde los significados intuitivos a sus respectivas formalizaciones. También, que se detallen procedimientos que ayuden a los estudiantes en el desarrollo de las actividades en las que se espera, puedan dominar las propiedades y características que definen a las figuras (2D y 3D) para posteriormente utilizarlas en la deducción y argumentación.

Con respecto al componente Argumentos, se sugiere promover y potenciar en el currículo la argumentación lógica de los resultados en la descripción y localización de objetos por parte de los estudiantes. Es importante que estos argumentos sean desarrollados a través de significados intuitivos e informales de los conceptos geométricos para realizar las justificaciones y conclusiones.

En el currículo, el componente Relaciones se encuentra presente al conectar algunas ideas con otros contenidos matemáticos (patrones, conjunto). Se sugiere promover la conexión de ideas geométricas, por ejemplo, que los estudiantes relacionen los atributos de las figuras $2 \mathrm{D}$ con los de las figuras 3D como también, la orientación y localización de objetos en situaciones de la vida cotidiana. Es importante también, dejar explícito que es relevante mostrar colecciones de una misma figura en diferen- 
tes posiciones y con diferentes medidas para no crear en los estudiantes los llamados prototipos (Hershkowitz, 1989). Así mismo, el trabajar la orientación y localización con objetos orientados y no orientados, permitirá el diálogo y la argumentación sobre los contenidos geométricos.

\section{Conclusiones}

Con la realización de este estudio se ha mostrado el uso de un instrumento en el análisis de uno de los Currículos Nacionales Interculturales Bilingües de Ecuador (Kichwa), lo que puede servir de orientación para su rediseño, su implementación y en la evaluación de la enseñanza sobre la geometría espacial.

Esta herramienta teórica y metodológica fue elaborada como un sistema de indicadores que no se considera definitivo ni cerrado y como una herramienta que "el profesor de matemática debe conocer, comprender y valorar...y adquirir competencia para su uso pertinente" (Godino, Batanero, Font y Giacomone, 2016, p. 282). Por lo que puede ser utilizada como pauta o guía por el profesorado para la reflexión sobre las características de un proceso de estudio planificado o implementado y de sus prácticas pedagógicas, como también en este caso, para la reflexión de un proceso de estudio planificado con la finalidad de lograr mejoras progresivas en ellos.

Su aplicación arrojó debilidades en cada componente de la faceta epistémica adaptada, motivo por el que se han propuesto sugerencias para el logro de un proceso de instrucción idóneo y la mejora del aprendizaje.

\section{Referencias}

Bisquerra, R. (1989). Métodos de investigación educativa. Barcelona: P.P.U.

Cruz A., Gea M. y Giacomone B. (2017). Criterios de idoneidad epistémica para el estudio de la geometría espacial en educación primaria. En J. M. Contreras, P. Arteaga, G. R. Cañadas, M. M. Gea, B. Giacomone y M. M. López-Martín (Eds.), Actas del Segundo Congreso Internacional Virtual sobre el Enfoque Ontosemiótico del Conocimiento y la Instrucción Matemáticos (pp. 1-10). Granada: UGR. Disponible en, enfoqueontosemiotico.ugr.es/civeos.html

Godino, J. D. (2013). Indicadores de la idoneidad didáctica de procesos de enseñanza y aprendizaje de las matemáticas. Cuadernos de Investigación y Formación en Educación Matemática, 11, 111-132.

Godino, J.D., Batanero, C., Font, V. y Giacomone, B. (2016). Articulando conocimientos y competencias del profesor de matemáticas: el modelo CCDM. En C. Fernández et al. (Eds.), Investigación en Educación Matemática XIX (pp. 272285). Málaga: SEIEM.

Hershkowitz, R. (1989), "Visualization in Geometry. Two Sides of the Coin", Focus on Learning Problems in Mathematics, 11(1), 61-76.

Ministerio de Educación (2017). Currículo Intercultural Bilingüe Kichwa. Quit 